\title{
Is There a Risk Factor More Responsible for Disaster?
}

\author{
Carasca Cosmin ${ }^{*}$, Muresan Vasile Adrian², Tilea Ioan³, Magdas Annamaria ${ }^{4}$, Carasca Emilian ${ }^{4}$, Incze \\ Alexandru 4 \\ ${ }^{1}$ County Emergency Clinical Hospital, Tirgu Mures, Romania \\ 2 Surgical Clinic 1, County Emergency Clinical Hospital, Tirgu Mures, Romania \\ 3 Cardiovascular Rehabilitation Clinic, Dept. Family Medicine, Institute of Cardiovascular Diseases and Transplantation Tirgu Mures, Romania, \\ 4 Internal Medical Clinic 4, County Clinical Hospital, Tirgu Mures, Romania
}

Background: Risk factors for peripheral arterial disease are generally the same as those responsible for the ischemic heart disease and in both cases are overlapping risk factors involved in the etiology of atherosclerosis, such as smoking, dyslipidemia, diabetes and hypertension. Case report: We present a case of a 61 years old male, whose ischemic peripheral symptoms began in 2003, at the age of 49, presenting as a Leriche syndrome. The patient was subjected to first revascularization procedure consisting in aortic-bifemoral grafting in the same year. General examination revealed no risk factors except smoking. Only a year after, he returns with critical right lower limb ischemia due to bypass thrombosis, therefore two thrombectomies were performed followed by a right side femoro-popliteal bypassing with Dacron prosthesis. The patient's condition was good until 2008 when a femoro-popliteal bypass using inverted autologus saphenous vein was imposed due to occlusion of the previous graft. In 2013 the patient was readmitted to hospital with left lower limb critical ischemia. A femoro-popliteal bypass was performed, followed by two thrombectomies and the amputation of the left thigh. Up to this date, the patient kept smoking.

Discussions: Although our patient has a low/medium risk level of atherosclerosis by Framingham score and a minimum Prevent III score, all the surgical revascularization procedures were not able to avoid the amputation.

Conclusions: There are enough reasons to believe that smoking as a single risk factor can strongly influence the unfavorable progression to amputation in patients with peripheral arterial disease.

Keywords: peripheral arterial disease, smoking, amputation.

Received: 9 December 2014 / Accepted: 19 April 2015

\section{Introduction}

The past decade has witnessed the dissemination of many new pharmacologic, percutaneous and surgical approaches that can improve the well-being of those individuals who suffer of peripheral arterial disease. This is often associated with risk factors including cigarette smoking, diabetes, hypertension or dyslipidemia and patients have a high risk of future vascular events. Good medical management results in improved outcome and quality of life, but previous studies have documented sub-optimal treatment of risk factors $[1,2]$. Of these, the modifiable risk factors, smoking and hypertension, contribute most heavily to disease progression in the patient with peripheral artery disease.

\section{Case report}

We present the case of a male, heavy smoker, at the moment age 61, whose ischemic peripheral suffering begins at the age of 49 with clinical signs of Leriche syndrome, with progressive worsening within one year. He was subjected to first revascularization procedure consisting in bifemoral aortic bypassing in the year of 2003. Clinical and biological evaluation revealed no risk factors except smoking. The short term postoperative progress was favorable, however only a year after, the patient returned with ischemic symptoms located to the right lower limb. Thrombosis of the

* Correspondence to: Cosmin Carasca

E-mail: cosmincarasca@yahoo.com right aortic-femoral prosthetic branch was noted. Two thrombectomys were performed, being soon after succeeded by a right femoral-popliteal grafting using Dacron prosthesis. The patient's condition was good until the year of 2008, when a right side femoral-popliteal bypass with inverted autologus saphenous vein was imposed due to occlusion of the Dacron graft. Up to this date, the patient kept smoking and no other risk factors were identified. After another five years of continuing smoking the patient was readmitted to the hospital with left lower limb critical ischemia. Left aorto-femoral prosthetic branch occlusion was noted due to a poor infra-inguinal and infra-genicular run-off. A femoral-popliteal bypass procedure was performed, followed by two thrombectomies and leading to amputation of the left thigh by the end of 2013. General examination indicated elevated systolic blood pressure values $(160 \mathrm{mmHg})$ and also a high blood sugar level of $100-180 \mathrm{mg} \%$. The patient was diagnosed with type II diabetes recently identified, although measurements were carried out under surgical and infectious stress conditions. The patient is actively evaluated a year after the amputation and he presents a nocturnal pain in the remaining right limb. The clinical status shows the following values: blood pressure 120-140/70-80 $\mathrm{mmHg}$ (under Perindopril 5mg/ day treatment); a blood sugar level of $90-105 \mathrm{mg} / \mathrm{dl}$ (fasting - under no treatment); glycated hemoglobin 
(A1c) $6,4 \%$; total cholesterol $<200 \mathrm{mg} / \mathrm{dl}$; tryglicerides $<130 \mathrm{mg} / \mathrm{dl}$ (under Atrovastatin $20 \mathrm{mg} /$ day permanent treatment); inflammatory tests (erythrocyte sedimentation rate, C-reactive protein and fibrinogen level) within normal ranges and the renal function is preserved. Since 2003 and up to this date the patient is under an efficient anticoagulant and antipatelet treatment with Trombostop and Aspenter but he is still smoking. The ankle-brachial index test indicates a value of 0.28 while the aortography and the right side extremity arteriography describe: no significant stenosis of the infra-renal aorta; occlusion of the aorticbifemoral graft (figure 1); occlusion of the femoralpopliteal vein graft; no visualization of the common femoral artery and the initial part of the superficial femoral artery; the deep femoral artery (figure 2), the first segment of the popliteal artery above the knee join (figure 3) and the anterior tibial artery are visible and suitable for further revascularization procedures. The patient finally quits smoking but he rejects any attempt of other surgical interventions. Therefore he is subjected to the first course of 10 days treatment with Alprostadilum 2x20mg/day, during which the nocturnal pain disappears. Although there is no improvement in the objective tests (ankle-brachial index test and photoplethysmography), at the present date the patient has no ischemic rest symptoms or nocturnal pain.

\section{Discussions}

Of the risk factors, smoking is the key of the development and progression of chronic atherosclerotic arteriopathy of the lower limbs, with data related to doseeffect connection [3, 4]. The risk among population for smoking and intermittent claudication calculated for

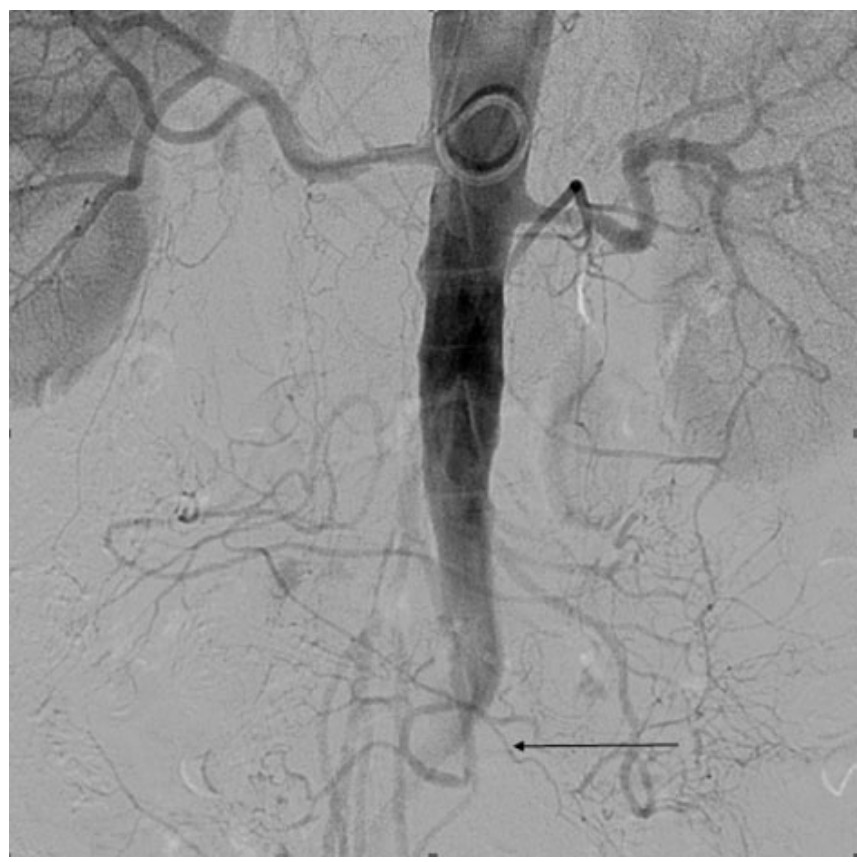

Fig. 1. Occlusion of the aortic-bifemoral graft - indicated by arrow

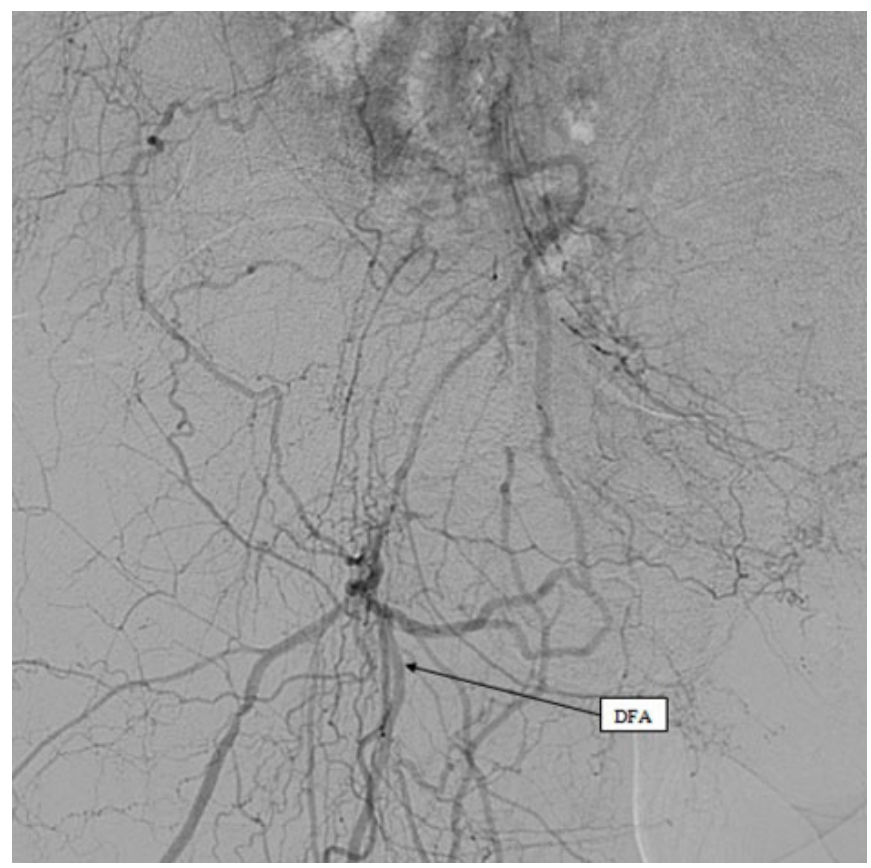

Fig. 2. Deep femoral artery (DFA) associated with a rich collateral circulation

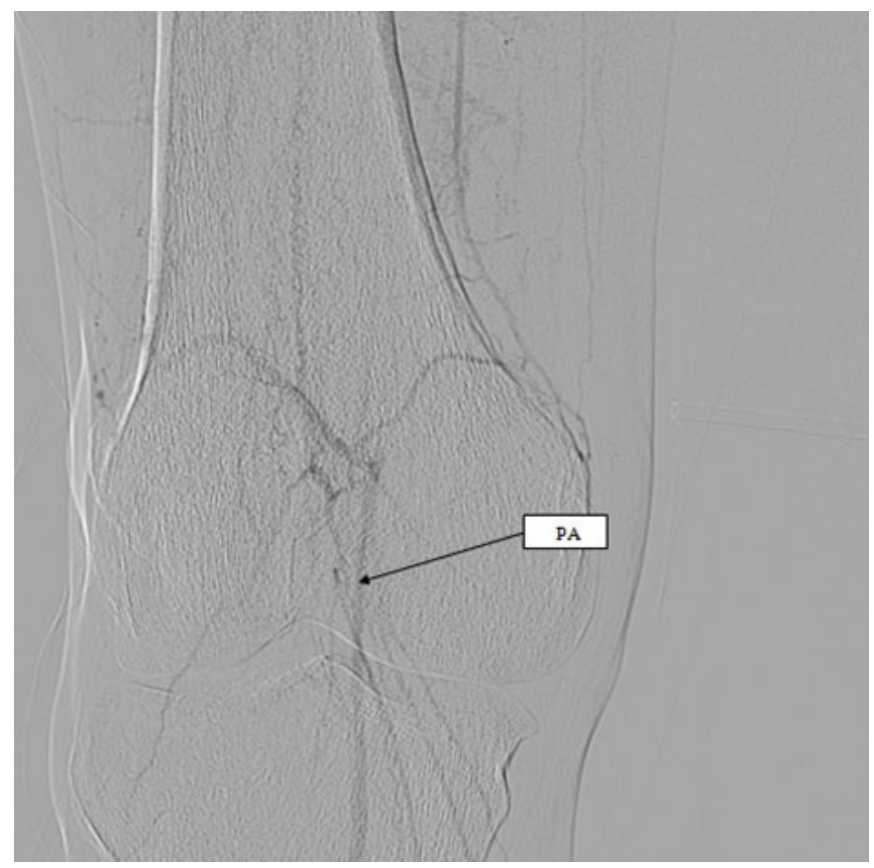

Fig. 3. Popliteal artery (PA) suitable for further revascularization procedures

cross-sectional studies ranges from $14 \%$ to $53 \%$ [5]. Smoking adversely affects postoperative graft patency and increases the risk of limb loss after surgical reconstruction [6-8]. The effectiveness of antiplatelet medications is diminished in smokers [9]. A few studies have reported that smoking cessation is associated with a rapid decline in the incidence of intermittent claudication [10] and the intermittent claudication risk for ex-smokers one year after quitting approximates that for nonsmokers $[10,11]$. In a referral population of claudicants without diabetes, smoking cessation was associated with a lack of progression to rest pain, a decreased surgical intervention rate, a decreased risk 
of cardiac events and improved survival [12]. In addition to retarding the incidence and progression of lower-extremity arterial disease and its associated risk of death, smoking cessation has been shown to decrease mortality risk after myocardial infarction [13]. In patients with peripheral arterial disease, smoking could represent a significant factor worsening the natural evolution of the disease. The cotinuation of the tobacco use is associated with a rapid acceleration of the disease progression, leading rapidly to critical limb ischemia and severe claudication, in the same time being associated with a lower longevity of the revascularization and a significant increase in the amputation rates. Continuation of smoking habit has also been proved to be associated with increase rates of acute myocardial infarction, stroke and death. Although our patient has a low/medium risk level of atherosclerosis by Framingham score and a minimum Prevent III score, the outcome of all surgical revascularization procedures only resulted in amputation. In all patients with peripheral arterial disease, immediate intervention is required for convincing them to stop smoking, focusing on how smoking cessation could be associated with a significant improvement in the quality of life and survival rates of these patients [14].

Another negative predictor, which is not to be neglected at all in this patient, is the immobilization at his home after the left thigh amputation carried out in October 2013 [15].

\section{Conclusions}

In patients with peripheral arterial disease, the first therapeutic gesture should be smoking cessation. Despite all efforts of revascularization in this case, the outcome of the multiple surgical procedures only resulted in limb loss. In particular, the use of evidence-based treatments is sub-optimal, while cigarette smoking is poorly managed. There are enough reasons to believe that smoking as a single risk factor can strongly influence the unfavorable progression to amputation.

\section{References}

1. Murabito JM, D'Agostino RB, Silbershatz H, Wilson PWF. Intermittent Claudication - A Risk Profile From The Framingham Heart Study. Circulation, 1997;96:44-49.

2. Khan S, Flather M, Mister R et al. Characteristics and Treatments of Patients with Peripheral Arterial Disease Referred to UK Vascular Clinics: Results of a Prospective Registry. European Journal of Vascular and Endovascular Surgery, 2007;33:4:442-450.

3. Bainton D, Sweetnam P, Baker I, Elwood P. Peripheral vascular disease: consequence for survival and association with risk factors in the Speedwell Prospective Heart Disease Study. Br Heart J, 1994;72:128132.

4. Bowlin SJ, Medalie JH, Flocke SA, Zyzanski SJ, Goldbourt U. Epidemiology of intermittent claudication in middle-aged men. Am J Epidemiol, 1994;140:418-430.

5. Fowkes FGR. Epidemiology of atherosclerotic arterial disease in the lower limbs. Eur J Vasc Surg, 1988;2:283-291.

6. Sayers RD, Thompson MM, Dunlop P, London NJ, Bell PR. The fate of infrainguinal PTFE grafts and an analysis of factors affecting outcome. Eur J Vasc Surg, 1994;8:607-610.

7. Ameli FM, Stein M, Provan JL, Prosser R. The effect of postoperative smoking on femoropopliteal bypass grafts. Ann Vasc Surg, 1989;3:2025.

8. Radack K, Wyderski RJ. Conservative management of intermittent claudication. Ann Intern Med, 1990;113:135-146.

9. Lepantalo M, Lassila R. Smoking and occlusive peripheral arterial disease: clinical review. Eur J Surg, 1991;157:83-87.

10. Ingolfsson $I O$, Sigurdsson $G$, Sigvaldason $H$, Thorgeirsson $G$, Sigfusson N. A marked decline in the prevalence and incidence of intermittent claudication in Icelandic men 1968-1986: a strong relationship to smoking and serum cholesterol-the Reykjavik Study. J Clin Epidemiol, 1994;47:1237-1243.

11. Dagenais GR, Maurice S, Robitaille NM, Gingras S, Lupien PJ. Intermittent claudication in Quebec men from 1974-1986: the Quebec Cardiovascular Study. Clin Invest Med, 1991;14:93-100.

12. Jonason T, Bergstrom R. Cessation of smoking in patients with intermittent claudication: effects on the risk of peripheral vascular complications, myocardial infarction and mortality. Acta Med Scand, 1987;221:253-260.

13. Voors AA, van Brussel BL, Plokker HWT et al. Smoking and cardiac events after venous coronary bypass surgery: a 15-year follow-up study. Circulation, 1996;93:42-47.

14. Kannel WB, Shurtleff D. The Framingham Study: cigarettes and the development of intermittent claudication. Geriatrics, 1973;28:61-68.

15. Hamburg NM, Balady GJ. Exercise rehabilitation in peripheral artery disease: functional impact and mechanisms of benefits. Circulation, 2011;123(1):87-97. 